\title{
Estilos de Aprendizagem e o Desenvolvimento do Estudante Adulto
}

\section{Learning Styles and Adult Student Development}

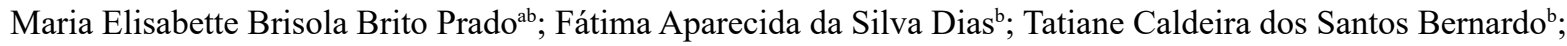 \\ Elisabete Alves Soares ${ }^{b}$
}

\begin{abstract}
aUniversidade Anhanguera de São Paulo, Programa de Pós-Graduação Stricto Sensu em Educação Matemática. SP. bUnopar, Programa de Pós-Graduação Stricto Sensu em Metodologias de Ensino de Linguagens e suas Tecnologias. PR, Brasil. *E-mail:
\end{abstract}

\begin{abstract}
Resumo
Este estudo tem como objetivo sistematizar aspectos relacionados à Teoria da Aprendizagem Experiencial de David Kolb no que tange aos Estilos de Aprendizagem, associando esta teoria com os estudos andragógicos. O estudante do Ensino Superior, numa primeira ou subsequente graduação, deve ser considerado um adulto aprendente que traz consigo uma bagagem cultural como estudante e/ou como profissional e, sobretudo, experiências, as quais podem servir de pontes entre o conhecimento prático vivido e o conhecimento técnico e teórico, resultando em aprendizagens significativas para o desenvolvimento de competências necessárias para lidar com os artefatos de uma sociedade tecnológica em contínua e rápida transformação.
\end{abstract}

Palavras-chave: Teoria Experimental. Autoaprendizagem. Ciclo de Aprendizagem.

\begin{abstract}
This study aims to systematize aspects related to David Kolb's Theory of Experiential Learning with respect to Learning Styles, associating this theory with andragogic studies. The student of Higher Education, in a first or subsequent graduation, must be considered an adult learner who brings with him a cultural background as a student and / or as a professional and, above all, experiences, which can serve as bridges between the practical knowledge lived and the technical and theoretical knowledge, resulting in significant learning for the development of skills necessary to deal with the artifacts of a technological society in continuous and rapid transformation.
\end{abstract}

Keywords: Experimental Theory. Self-learning. Learning Cycle.Keywords.

\section{Introdução}

Este artigo tem como objetivo apresentar um estudo teórico sobre os aspectos relacionados à Teoria da Aprendizagem Experiencial de Kolb (1984) no que tange aos Estilos de Aprendizagem, associando esta teoria com os estudos andragógicos. Esta temática se justifica por reconhecermos que independentemente do contexto no qual o estudante do Ensino Superior esteja situado, numa primeira ou subsequente graduação, deve ser considerado um adulto aprendente que traz consigo uma bagagem cultural como estudante e/ou como profissional e, sobretudo, experiências, as quais podem servir de pontes entre o conhecimento prático vivido e o conhecimento técnico e teórico, resultando em aprendizagens significativas para a vida. Aprender de forma significativa potencializa o estudante a desenvolver sua autonomia, o pensamento crítico, reflexivo e o autoestudo (heutagogia, autoaprendizagem), que segundo Hase e Kenyon (2000), incidem sobre o desenvolvimento de pessoas para lidar com um mundo em contínua e rápida transformação.

A Teoria da Aprendizagem Experiencial desenvolvida por David Kolb busca compreender como o estudante adulto aprende, se apropria do conhecimento, toma decisões e soluciona problemas. A partir dessas questões, o autor, com base em estudos científicos de renomados autores, a exemplo de Bruner, Flavell, Rogers, Singer, entre outros, define que aprendizagem emerge do processo de transformação da experiência, ou seja, resulta da combinação entre a vivência, compreensão e transformação. Contudo, destaca-se que não é qualquer experiência que resulta em aprendizagem, e sim que é necessária a reflexão consciente sobre as vivências que só então propiciam transformá-las em aprendizagens (KOLB, 1984).

\section{Desenvolvimento}

O processo de aprender é contínuo e ocorre ao longo da vida do estudante, que, motivado pelos próprios propósitos, empenha-se para aprender aquilo que lhe faz sentido, aquilo que lhe é significativo e reconhecidamente funcional para si. Consoante Da Silva, Sonaglio e Godoi (2013, p. 29-130), não são:

[...] esquemas conceituais arrojados e distantes da experiência real que alicerçariam a educação, mas sim a continuidade da experiência que é fundamental para a teoria da aprendizagem, defendendo que o processo vai durar enquanto durar a vida e a aprendizagem contínua. 
A teoria kolbiana se pauta na inter-relação entre características internas do homem e situações externas do ambiente, ou seja, entre o conhecimento de origem pessoal e social. Neste processo reflexivo, devem ser consideradas as várias dimensões que compõem o funcionamento psicológico do homem: a cognição, os sentimentos, emoções, percepção e ação.

A perspectiva do autor concebe o homem como um aprendiz que parte das suas próprias experiências, na reflexão e transformação das mesmas, por meio da dialética representada em sua teoria pelos pares "ação / reflexão" e "experiência / abstração", que compreenderemos mais adiante.

Esses aspectos dialogam com os estudos andragógicos, a medida que reconhecemos a experiência pessoal como um dos princípios determinantes no processo de aprendizagem de adultos. Knowles (1984), um dos grandes estudiosos da andragogia, amplamente referenciado na área, entende que a aprendizagem é pautada na experiência, motivação, foco, autodirecionamento, relevância para o estudante, bem como a compreensão da aplicabilidade do aprendizado.

A teoria experiencial está pautada justamente em subsidiar práticas que melhor se adequem ao processo de construção e sistematização do conhecimento de estudantes adultos ao identificar e descrever modelos e estilos de aprendizagem constituídos por traços peculiares e dominantes em cada estilo. Por estilo de aprendizagem, Kolb (1984, p. 24) define como sendo: "[...] um estado duradouro e estável que deriva de configurações consistentes das transações entre o indivíduo e o seu meio ambiente".

A caracterização dos estilos organizados na teoria kolbiana, todavia, não se constitui em rótulos estanques aos modos de aprender do estudante, apenas indicam o perfil preponderante nesse processo. Com a consciência sobre a predominância do estilo de aprendizagem do estudando, permite-se a personalização da aprendizagem e, assim, a possibilidade de desenvolveros demais estilos, o que favorece o chamado desenvolvimento integral da aprendizagem (KOLB, 1984).

É a este processo de desenvolvimento e aprimoramento dos estilos de aprendizagem que Kolb caracteriza como processo adaptativo da aprendizagem. Mas, antes de compreender a potencialidade de desenvolvimento destes estilos, é preciso, em primeiro lugar, compreender de que forma é estruturada a perspectiva do autor.

Pautado em pesquisas sobre o desenvolvimento do conhecimento e do pensamento, Kolb (1984) contemplou quatro dimensões estruturais no constructo teórico da Aprendizagem Experiencial: estrutura afetiva; estrutura perceptual; estrutura simbólica e estrutura comportamental.

Baseada em pensamentos de teóricos basilares da educação, Kolb caracteriza o processo de aprendizagem como holístico, que significa que sua base se fundamenta no contextualismo, uma vez que trata a aprendizagem como um processo no qual estudante e acontecimento desenvolvem-se simultaneamente.

Diante disso, é importante dizer que esta teoria não pretende situar os alunos em determinações ou mesmo classificações exatas sobre seus estilos de aprendizagem, ou seja, não tratase de uma definição imutável ou inerente ao ser (INÊS, 2009). A proposta do contextualismo é ver o ser humano sob um olhar justamente da individualidade,ou seja, a partir de uma ótica que busca uma aprendizagem com base na experiência e que possa proporcionar um modelo multidimensional de aprendizado para adultos (KOLB; KOLB, 2013).

A partir desta visão, Kolb (1984) situa preposições que guiam sua teoria, sistematizadas no Quadro 1.

Quadro 1 - Proposições norteadoras da Teoria da Aprendizagem Experiencial

\begin{tabular}{|l|l|}
\hline \multicolumn{1}{|c|}{$\begin{array}{c}\text { Proposições } \\
\text { Basilares }\end{array}$} & \multicolumn{1}{|c|}{ Integração na Teoria } \\
\hline $\begin{array}{l}\text { Aprendizagem } \\
\text { é processo }\end{array}$ & $\begin{array}{l}\text { A aprendizagem não pode ter como foco } \\
\text { um resultado a ser alcançado, mas sim o } \\
\text { aproveitamento do desenvolvimento. }\end{array}$ \\
\hline $\begin{array}{l}\text { Aprender é } \\
\text { reaprender }\end{array}$ & $\begin{array}{l}\text { Parte do princípio construtivista de que } \\
\text { é } \\
\text { conheciso experimentar a construção do de modo que ao vivenciá-la } \\
\text { reaprende-se constantemente. }\end{array}$ \\
\hline $\begin{array}{l}\text { Aprender } \\
\text { é resolver } \\
\text { conflitos }\end{array}$ & $\begin{array}{l}\text { Os conflitos permitem a reflexão, tomada de } \\
\text { decisões e elaboração de novas ações. }\end{array}$ \\
\hline $\begin{array}{l}\text { Aprender é } \\
\text { adaptar-se }\end{array}$ & $\begin{array}{l}\text { A constante tentativa de adaptação e } \\
\text { readaptação do indivíduo permite compreender } \\
\text { que aprendizagem não se trata somente de } \\
\text { cognição, mas também de um processo de } \\
\text { sentir, perceber e, sobretudo, de ser (atitude, } \\
\text { comportamento). }\end{array}$ \\
\hline $\begin{array}{l}\text { Resultados } \\
\text { vêm da } \\
\text { interação } \\
\text { indivíduo- } \\
\text { mundo }\end{array}$ & $\begin{array}{l}\text { Como mencionado no item anterior, aprender } \\
\text { é também adaptar-se e a ideia de comportar- } \\
\text { se em determinado ambiente está diretamente } \\
\text { ligada à relação do indivíduo com o ambiente } \\
\text { experimentado. }\end{array}$ \\
\hline $\begin{array}{l}\text { Aprender é } \\
\text { um processo } \\
\text { criativo }\end{array}$ & $\begin{array}{l}\text { Parte-se do pressuposto que a criatividade } \\
\text { emerge da associação entre conhecimento } \\
\text { pessoal e social, ou seja, da relação entre o } \\
\text { conhecido e o novo possível }\end{array}$ \\
\hline Fonte: Baseado
\end{tabular}
Fonte: Baseado em Kolb (1984).

Kolb (1984) propôs modelos explicativos, cuja organização e funcionamento definem os diferentes estilos de aprendizagem, constituídos por quatro modelos adaptativos de aprendizagem que se conjugam de forma cíclica, conforme apresentamos na sequência. Neste ciclo, o estudante/aprendiz vivencia:

Experiência Concreta (EC), que se refere ao contato direto com a situação e, portanto, envolve o fazer e experienciar, sendo que esse fazer se pauta inicialmente em conhecimentos já existentes.

Observação Reflexiva (OR), caracteriza-se em um movimento voltado para o interior, pela reflexão sobre a ação realizada, buscando identificar seus efeitos e as principais relações.

Conceituação Abstrata (CA), constitui-se pela formação de conceitos abstratos sendo pela generalização de regras e 
princípios, assim como, pela generalização decorrente dos elementos da experiência refletidos.

Experiência Ativa (EA), caracteriza-se em um movimento voltado para o externo, pela aplicação prática dos conhecimentos e processos de pensamento tornados refletidos, explicados e generalizados.

Quando mencionamos anteriormente a ciclicidade existente entre estes modelos adaptativos de aprendizagem, condicionamo-nos a dizer que é recursivo, ou seja, "sensível à situação de aprendizagem e ao que está sendo aprendido" (KOLB; KOLB, 2013, p. 8), como representado na Figura 1.

Figura 1 - O Ciclo de Aprendizagem Experiencial

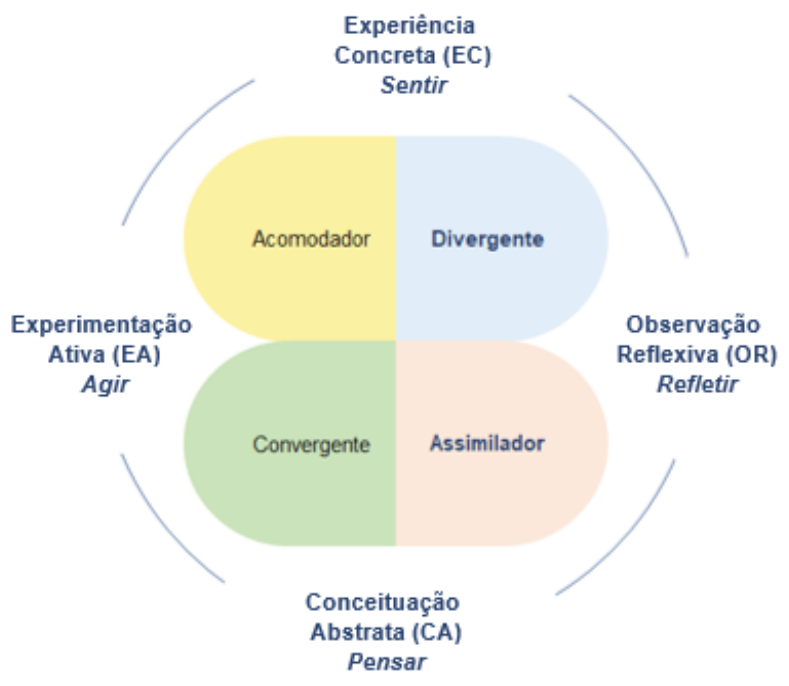

Fonte: Adaptado de Kolb e Kolb (2013).

O ciclo acima representado foi estruturado com base na sistematização das ideias de Kolb (1984) para que possamos compreender como a definição dos estilos de aprendizagem funciona. É possível observar na figura, o modelo de Kolb trabalha

como um instrumento para identificação individual do estilo de aprendizagem (Inventário de Estilos de Aprendizagem), o qual é aplicado neste estudo. A partir da classificação atribuída para cada sentença, o inventário mede a ênfase de cada estudante para cada um dos quatro modelos adaptativos de aprendizagem (EC, OR, CA e EA).

Kolb (1984) explica porque, então, define esta relação como dialética, uma vez que o estudante pode indicar prioridade à abstração sobre a concretude (pensamento / sentimento) e a experimentação ativa sobre a reflexão (ação / reflexão). A relação dialógica existente entre sentir e pensar demonstra como o estudante percebe a informação (CA-EC), e ainda a relação entre a reflexão e a ação definem como o estudante processa esta informação (EA-OR).

Temos assim, então, estudantes que apresentam dominâncias sobre estes modelos. Por exemplo, um estudante bastante observador, que comumente elabora teorias e hipóteses sobre um determinado conteúdo estudado, mas que não costuma aplicar os pensamentos que elabora, localizariase na Figura 1 entre os modelos OR e CA. A este estudante, definiríamos o estilo de aprendizagem Assimilador.

Portanto, quando combinados entre si, os modelos adaptativos de aprendizagem geram os, então, estilos de aprendizagem, que foram sistematizados em suas características no Quadro 2.

Quadro 2 - Os Estilos de Aprendizagem de David Kolb

\begin{tabular}{|l|c|}
\hline \multicolumn{1}{|c|}{ Estilos } & $\begin{array}{c}\text { Habilidades } \\
\text { dominantes }\end{array}$ \\
\hline $\begin{array}{l}\text { Divergente - aprende experimentando, } \\
\text { criando ideias e teorias, observando e } \\
\text { escutando, e relacionando o conteúdo do } \\
\text { curso com sua vivência. Capaz de analisar } \\
\text { as situações em diferentes pontos de vistas e } \\
\text { relacioná-las num todo organizado. }\end{array}$ & $\begin{array}{c}\text { Experiência } \\
\text { Concreta e } \\
\text { Observação } \\
\text { Reflexiva } \\
\text { EC - OR }\end{array}$ \\
$\begin{array}{l}\text { Assimilador - aprende refletindo, ouvindo, } \\
\text { observando e criando teorias e ideias, } \\
\text { preocupa-se pouco com suas aplicações. }\end{array}$ & $\begin{array}{c}\text { Conceituação } \\
\text { Abstrata e } \\
\text { Observação } \\
\text { Reflexiva } \\
\text { CA-OR }\end{array}$ \\
\hline $\begin{array}{l}\text { Convergente - aprende por ensaio e erro, e } \\
\text { por aplicação prática de ideias e teorias. É } \\
\text { hábil para definir problemas e para tomar } \\
\text { decisões. Utiliza raciocínio dedutivo. }\end{array}$ & $\begin{array}{c}\text { Conceituação } \\
\text { Abstrata e } \\
\text { Experimentação } \\
\text { Ativa } \\
\text { CA - EA }\end{array}$ \\
\hline $\begin{array}{l}\text { Acomodador - aprende por meio da } \\
\text { experiência e aplicação do conhecimento em } \\
\text { situações novas de aprendizagem. }\end{array}$ & $\begin{array}{c}\text { Experiência } \\
\text { Concreta e } \\
\text { Experimentação } \\
\text { Ativa }\end{array}$ \\
\hline Fonte: Kolb (1984). & EC - EA \\
\hline
\end{tabular}

Na representação da Figura 1, para representarmos o estilo de aprendizagem de um estudante, então, ao desenharmos nele suas preferências o quadrante passava a formar um desenho similar a uma "pipa", ou seja, caracterizando a preferência relativa do indivíduo dentre as quatro fases do ciclo de aprendizagem e aquela que fosse preponderante caracterizava, então, o estilo de aprendizagem individual daquele estudante.

Vários são os fatores que podem definir os estilos de aprendizagem dos estudantes. A composição genética, as experiências individuais e o contexto sócio-histórico constituem-se como fatores determinantes à predisposição por um destes estilos de aprendizagem. Assim, o estilo de aprendizagem individual segue uma combinação única de preferências e até mesmo de personalidade. Para melhor definir esta questão, Kolb (1984) organizou os estilos de aprendizagem conforme o nível de comportamento do estudante de acordo com suas características nas seguintes áreas: tipo de personalidade, área de conhecimento, carreira profissional, trabalhos atuais e competências adaptativas. Cada uma destas áreas destaca características comuns para cada estilo de aprendizagem.

Por fim, de modo a completar a compreensão do quadrante organizado por Kolb (2013) devemos compreender porque, então, o autor trata estes modelos como adaptativos e como um processo cíclico. Um exemplo seria uma pessoa com um tipo de personalidade introvertida, mas intuitiva, que se enquadraria mais no tipo Assimilador. Contudo, apontar 
estas características predominantes nesta área não significa dizer que um estudante, predominantemente Assimilador, por exemplo, não possa ter qualquer outro estilo de aprendizagem em outras áreas, pelo contrário. Com isso, o autor elucida que um estudante pode se adaptar a novos estilos de aprendizagem e, inclusive, esta adaptação, demonstra a evolução dos indivíduos no ciclo, determinados pelas suas preferências nos modos de aprendizagem - EC, OR, CA e EA.

Todas estas articulações estão na base do processo de aprendizagem postulado por Kolb: apreender e compreender fundamentam a construção de conhecimentos concretos e abstratos da mesma forma que intenção e extensão constituem os processos de transformação, basilares do desenvolvimento da aprendizagem de Kolb. A compreensão a respeito deste processo de transformação e desenvolvimento, de acordo com Cerqueira (2000), conforme o nível de desenvolvimento do indivíduo avança, mais integrativa o estudante é capaz de tornar suas dimensões de aprendizagem.

Em suma, "[...] cada indivíduo tem seu estilo de aprendizagem e que o mesmo poderá desenvolver outro estilo, desde que estimulado [...] o desenvolvimento de novas habilidades na formação do discente [...]" (DA SILVA; GODOI; SONAGLIO, 2013, p. 124-125). Isso nos leva a questionar como deve ser desenvolvido o papel do professor/ tutor neste contexto.

Consoante Almeida (2010), os docentes precisam elaborar aulas que explorem os estilos de aprendizagem dominantes dos estudantes, de modo que possam explorar suas preferências, mas também devem dar condições e possibilidades de que estes estudantes possam desenvolver aqueles que não são preferenciais. De acordo com Da Silva, Godoi e Sonaglio (2013), estes novos estilos podem ser incentivados pelos professores/tutores de modo que os estudantes possam sempre desenvolver-se mais em suas habilidades, mas principalmente desenvolver seu autoconhecimento enquanto seresaprendentes, compreendendo suas características e buscando trabalhar outras que podem ainda ser desenvolvidas.

Essa consciência do estudante sobre si e o reconhecimento de suas experiências como fontes de conhecimento é uma das condições que, aliadas às tecnologias digitais da informação e comunicação, potencializa o desenvolvimento de atitudes autônomas focadas na autoaprendizagem, no sentido dado por Hase e Kenyon (2000), sobre a heutagogia ${ }^{1}$.

De acordo com as autoras, os caminhos traçados pelos estudantes no desenvolvimento de sua aprendizagem recaíam, em grande parte, sobre o papel dos professores/ tutores, perspectiva que tem se modificado nos últimos 30 anos ou mais, haja vista que pudemos compreender mais aprofundadamente sobre como os alunos aprendem, como podem ser protagonistas neste percurso e, ainda, partindo da compreensão de que proporcionado um ambiente adequado, os estudantes terão maiores condições de aprender e serem, então, autodirigidos sobre como o aprendizado é aplicado (HASE; KENYON, 2000).

A heutagogia é, portanto, uma teoria que olha para o futuro e para estas novas habilidades que professores e tutores devem se atentar para potencializar em seus estudantes, uma vez que reconhece que saber aprender será uma habilidade essencial do estudante, considerando o ritmo dado pelas tecnologias e inovações que têm modificado tanto as estruturas de trabalho quanto das comunidades (HASE; KENYON, 2000).

\section{Conclusão}

Considerando a possibilidade do desenvolvimento da autoaprendizagem, do estudante em aprender a aprender com autonomia e de forma significativa para vida, a qual requer cada vez mais um processo dinâmico e rápido de aprender, investigar, criar e recriar produtos e conhecimentos é que o papel do professor/tutor precisa ser revisto, para assumir uma postura de mediador, orientador, mentor do estudante nestas definições sobre as quais os próprios estudantes muitas vezes não têm conhecimento.

\section{Referências}

ALMEIDA, K.R. Descrição e análise de diferentes estilos de aprendizagem. Interlocução, v.3, n.3, p.38-49, 2010.

CERQUEIRA, T.C.S. Estilos de aprendizagem em universitários. Campinas: Unicamp, 2000.

HASE, S.; KENYON, C. From Andragogy to Heutagogy. Austrália: Southem Cross University, 2000.

KNOWLES, M. Self-Directed Learning: a guide for learners and teachers. Chicago: Follett Publishing Company, 1975.

KNOWLES, M. S. The modern practice of adult education: from pedagogy to Andragogy. Chicago: Follet, 1984.

KOLB, A.Y.; KOLB, D. The kolb learning style inventory 4.0: a comprehensive guide to the theory, psychometrics, research on validity and educational applications. Philadelphia: HayGroup, 2013.

KOLB, D.A. Experiential learning: experience as the source of learning and development. New Jersey: Prentice-Hall, 1984.

SONAGLIO, A.L.B.; GODOI, C. K.; DA SILVA, A. B. Estilos de aprendizagem experiencial e aquisição de habilidades: um estudo com discentes de graduação em administração em instituições de ensino superior. Adm. Ensino Pesq., v.14, n.1, p.123-159, 2013.

INÊS, R.P.R. A aprendizagem experiencial e a sabedoria no adulto e no adulto idoso. Lisboa: Universidade de Lisboa, 2009. 